\title{
Clinical utility gene card for: osteogenesis imperfecta
}

\author{
Fleur S van Dijk ${ }^{\star, 1}$, Raymond Dalgleish ${ }^{2}$, Fransiska Malfait ${ }^{3}$, Alessandra Maugeri ${ }^{1}$, Agnieszka Rusinska $^{4}$, \\ Oliver Semler ${ }^{5}$, Sofie Symoens ${ }^{3}$ and Gerard Pals ${ }^{1}$
}

European Journal of Human Genetics (2013) 21, doi:10.1038/ejhg.2012.210; published online 26 September 2012

\section{DISEASE CHARACTERISTICS}

1.1 Name of the disease (synonyms)

Osteogenesis imperfecta (OI). OI types I-V..$^{1-3}$ OI types VI-XII. ${ }^{4}$

\subsection{OMIM\# of the disease}

\#166200 (type I), \#166210 (type II), \#259420 (type III), \#166220 (type IV), \%610967 (type V), \#613982 (type VI), \#610682 (type VII), \#610915 (type VIII), \#259440 (type IX), \#613848 (type X), \#610968 (type XI), \#613849 (type XII).

\subsection{Name of the analysed genes or DNA/chromosome segments}

\begin{tabular}{lllc}
\hline Gene & Name & Locus & Inheritance \\
\hline BMP1 & Bone morphogenetic protein 1 & $8 \mathrm{p} 21.3$ & $\mathrm{AR}$ \\
COL1A1 & Collagen, type I, alpha 1 & $17 \mathrm{q} 21.33$ & $\mathrm{AD}$ \\
COL1A2 & Collagen, type I, alpha 2 & $7 \mathrm{q} 22.3$ & $\mathrm{AD}$ \\
CRTAP & Cartilage associated protein & $3 \mathrm{p} 22.3$ & $\mathrm{AR}$ \\
FKBP10 & FK506 binding protein 10, 65 kDa & $17 \mathrm{q} 21.2$ & $\mathrm{AR}$ \\
IFITM5 & Interferon induced transmembrane protein 5 & $11 \mathrm{p} 15.5$ & $\mathrm{AD}$ \\
LEPRE1 & Leucine proline-enriched proteoglycan & $1 \mathrm{p} 34.2$ & $\mathrm{AR}$ \\
& (leprecan) 1 & & \\
PLOD2 & Procollagen-lysine, 2-oxoglutarate & $3 \mathrm{q} 24$ & $\mathrm{AR}$ \\
& 5-dioxygenase 2 & & \\
PPIB & Peptidylprolyl isomerase B (cyclophilin B) & $15 \mathrm{q} 22.31$ & $\mathrm{AR}$ \\
SERPINF1 & Serpin peptidase inhibitor, clade F & $17 \mathrm{p} 13.3$ & $\mathrm{AR}$ \\
& (alpha-2 antiplasmin, pigment epithelium & & \\
& derived factor), member 1 & & \\
SERPINH1 & Serpin peptidase inhibitor, clade H & $11 \mathrm{q} 13.5$ & $\mathrm{AR}$ \\
& (heat shock protein 47), member 1, & & \\
& (collagen binding protein 1) & & \\
SP7 & Sp7 transcription factor & 12q13.13 & AR \\
\hline
\end{tabular}

aThe gene names are written according to the HUGO Gene Nomenclature Commitee website (http://www.genenames.org).

bThe loci are written according to the UCSC genome browser (http://genome.ucsc.edu/) using The loci are written according to the UCSC gen
the current genome assembly (GRCh37/hg19).

${ }^{\mathrm{C}} \mathrm{AD}=$ autosomal dominant, $\mathrm{AR}=$ autosomal recessive.

dThe vast majority of COL1A2 mutations are autosomal dominantly inherited, but there is one which is nominally recessive: c. $3105+2 \mathrm{~T}>\mathrm{C}$.

1.4 OMIM\# of the gene(s)

BMP1 (*112264), COL1A1 (+ 120150), COL1A2 $\left({ }^{*} 120160\right)$, CRTAP $\left({ }^{*} 605497\right)$, FKBP10 $\left({ }^{\star} 607063\right)$, LEPRE1 $\left({ }^{*} 610339\right)$, PLOD2 $\left({ }^{\star} 601865\right)$, PPIB $\left({ }^{\star} 123841\right)$, SERPINF1 $\left({ }^{\star} 172860\right)$, SERPINH1 $\left({ }^{*} 600943\right)$, SP7 $\left({ }^{\star} 606633\right)$, IFITM5 $\left({ }^{*} 614757\right)$.

\subsection{Mutational spectrum}

\subsubsection{Dominant OI}

Osteogenesis imperfecta comprises a heterogeneous group of diseases characterized by susceptibility to bone fractures, specific extraskeletal manifestations and in most cases with presumed or proven defects in collagen type I biosynthesis. Recently, the International Nomenclature Committee for Constitutional Disorders of the Skeleton proposed to classify OI into five types (OI types I-V), based on distinct clinical/ radiological characteristics. ${ }^{3}$ In OI types I-IV, more than 1000 different variants in the COL1A1 and COL1A2 genes have been identified (Dalgleish, R: Osteogenesis Imperfecta Variant Database https://oi.gene.le.ac.uk, accessed 16 May 2012). ${ }^{4,5}$ The genetic cause of OI type V has recently been discovered and consists of a single recurrent 5'UTR mutation c.-14C $>\mathrm{T}$ in the IFITM5 gene. ${ }^{6,7}$ Approximately $90 \%$ of all cases are the result of a causative variant in either the COL1A1 or COL1A2 gene. Some genotype-phenotype correlations exist. OI type I usually results from frameshift, nonsense and splice-site alterations in one COL1A1 allele that lead to mRNA instability and haploinsufficiency. In a low percentage of OI type I patients, substitutions for glycine by small amino acids (cysteine, alanine and serine) near the amino terminal ends of the triple helical domains encoded by either COL1A1 or COL1A2 are found. In contrast, OI types II, III and IV are usually caused by sequence variants in either COL1A1 or COL1A2 that result in substitutions for glycine residues in the uninterrupted Gly-X-Y triplet repeat of the 1014-residue triple-helical domains encoded by each gene. Less common causative variants include splice site alterations, variants in the carboxyl-terminal propeptide coding domains, or insertion/deletion events that lead to in-frame sequence alterations. ${ }^{8}$

\subsubsection{Recessive $O I$}

Recently, many genetic causes of recessive OI have been elucidated. ${ }^{8,9}$ Recessive mutations in the nine currently known genes cause a clinical/radiological phenotype comparable to or even indistinguishable from OI type II, III or IV caused by COL1A1 or COL1A2 mutations. ${ }^{10}$ However, as the underlying genetic defect is different, some investigators promote an extended classification of OI with addition of new types (OI types VI-XIII) solely based upon a different genetic defect. ${ }^{11}$ The causative variants found in the recessive genes (Dalgleish, R: Osteogenesis Imperfecta Variant Database https:// oi.gene.le.ac.uk, accessed 16 May 2012), ${ }^{4,5}$ are typically homozygous or compound heterozygous loss-of-function mutations that result in a null allele with severely decreased or no production of normal protein.

\footnotetext{
${ }^{1}$ Department of Clinical Genetics, Center for Connective Tissue Disorders, VU University Medical Center, Amsterdam, The Netherlands; ${ }^{2}$ Department of Genetics, University of Leicester, Leicester, UK; ${ }^{3}$ Center for Medical Genetics, Ghent University and Ghent University Hospital, Ghent, Belgium; ${ }^{4}$ Department of Paediatric Propedeutics and Bone Metabolic Diseases, Medical University of Lodz, Lodz, Poland; ${ }^{5}$ Children's Hospital, University of Cologne, Cologne, Germany

*Correspondence: Dr FS van Dijk, Department of Clinical Genetics, Center for Connective Tissue Disorders, VU University Medical Center, Amsterdam, MB 1007, The Netherlands. Tel: +1 3120 440150; Fax: +1 3120 4440769; E-mail: fs.vandijk2@vumc.nl or cct@vumc.nl
} 


\subsection{Analytical methods}

The first step is to perform sequence analysis of the COL1A1 and COL1A2 genes on genomic DNA (gDNA). If no causative variant is identified, the next step is to determine whether a deletion or duplication of some or all of the coding regions of either gene has occurred. This can be investigated with quantitative real-time PCR (qPCR), Multiplex ligation-dependent probe amplification (MLPA) or array-based analysis. If no mutation is identified, the clinical diagnosis of OI should be clinically re-evaluated. If the clinical diagnosis of OI is valid, gDNA analysis of the recessive genes, preferentially simultaneously, should be initiated. If no causative variants or only one causative variant is found, QPCR, MLPA or arraybased analysis is advised. ${ }^{8}$

\subsection{Analytical validation}

Sequence alterations that could be causative variants are bidirectionally sequenced, further investigated by in silico analysis and, if possible, by segregation analysis. Furthermore, identified variants should be checked against existing entries in the Osteogenesis Imperfecta Variant Database and SNP databases. In some cases, protein studies and mRNA/cDNA analysis isolated from cultured fibroblasts, can have an additive value as (i) the effect of unclassified variants suspected to alter splicing as well as (ii) the possible existence of deep intronic variants can be studied. ${ }^{8}$

\subsection{Estimated frequency of the disease}

(Incidence at birth ('birth prevalence') or population prevalence) OI has a birth prevalence of approximately 6-7/100.000. The prevalence and incidence of the OI types $\mathrm{I}-\mathrm{V}$ differ from each other with OI type I and OI type IV accounting for more than half of all OI cases. $^{12}$

\subsection{If applicable, prevalence in the ethnic group of investigated person}

As the dominantly inherited severe OI types (not OI type I) arise most often from de novo mutations in the COL1A1 and COL1A2 genes, a higher prevalence in particular ethnic groups is not expected. With regard to OI type I: most of the characterized cases are in persons of white European descent and, as such, it is not really possible to comment on whether or not differences in ethnic frequencies exist. One could argue that recessive OI would occur more frequently in inbred populations but this has not been properly studied. However, it is reported that a founder mutation in LEPRE1 is carried by $1.5 \%$ of West Africans and $0.4 \%$ of African Americans. ${ }^{13}$

\subsection{Diagnostic setting}

A. (Differential) diagnostics

B. Predictive testing

C. Risk assessment in relatives

D. Prenatal

$\begin{array}{ll}\text { Yes } & \text { No } \\ \otimes & \square \\ \otimes & \square \\ \otimes & \square \\ \otimes & \square\end{array}$

Comment: DNA analysis in OI is used mainly to confirm the clinical diagnosis. OI types II-V are more easy to diagnose clinically as they usually concern multiple (prenatal) fractures with deformation of bone and specific extraskeletal features (blue sclerae, short length, dentinogenesis imperfecta, hearing loss, early osteoporosis and joint laxity). OI type I can be more difficult to diagnose clinically because the spectrum can extend to nearly asymptomatic individuals with a mild predisposition to fractures, normal stature and normal lifespan ${ }^{14}$ as is observed in individuals with idiopathic juvenile osteoporosis. OI type I or sometimes IV are important differential diagnostic considerations in case of suspicion of non-accidental injury (NAI).

Predictive testing without clinical symptoms of OI is less common but does occur in specific circumstances. Genetic testing is necessary for genetic risk assessment in relatives. Pre-natal and pre-implantation genetic diagnosis is available for families with a known genetic cause of OI.

\section{TEST CHARACTERISTICS}

\begin{tabular}{|c|c|c|c|c|}
\hline & \multicolumn{2}{|c|}{ Genotype or disease } & \multirow{2}{*}{$\begin{array}{l}\text { A: True positives } \\
\text { B: False positives }\end{array}$} & \multirow{2}{*}{$\begin{array}{l}\text { C: False negatives } \\
\text { D: True negatives }\end{array}$} \\
\hline & Present & Absent & & \\
\hline \multicolumn{5}{|l|}{ Test } \\
\hline \multirow[t]{2}{*}{ Positive } & $A$ & B & Sensitivity: & $A /(A+C)$ \\
\hline & & & Specificity: & $D /(D+B)$ \\
\hline \multirow[t]{2}{*}{ Negative } & $\mathrm{C}$ & $\mathrm{D}$ & Positive predictive value: & $A /(A+B)$ \\
\hline & & & Negative predictive value: & $D /(C+D)$ \\
\hline
\end{tabular}

\subsection{Analytical sensitivity}

(proportion of positive tests if the genotype is present)

Genomic sequencing, if necessary combined with quantitative analysis (MLPA/qPCR/array-based analysis) and in some cases cDNA/mRNA or protein analysis approaches $100 \%$ for mutation detection.

\subsection{Analytical specificity} (proportion of negative tests if the genotype is not present) Analytical specificity is nearly $100 \%$. False positives in genomic sequencing are rare.

\subsection{Clinical sensitivity}

(proportion of positive tests if the disease is present)

The clinical sensitivity can be dependent on variable factors such as age or family history. In such cases a general statement should be given, even if a quantification can only be made case by case.

Genomic DNA sequencing of the known genes should identify causative variants in more than $95 \%$ of individuals with clinically confirmed OI in most populations. ${ }^{8}$

\subsection{Clinical specificity}

(proportion of negative tests if the disease is not present)

The clinical specificity can be dependent on variable factors such as age or family history. In such cases a general statement should be given, even if a quantification can only be made case by case.

Clinical specificity is nearly $100 \%$.

\subsection{Positive clinical predictive value} (life time risk to develop the disease if the test is positive) For OI inherited both as an autosomal dominant or as an autosomal recessive trait, non-penetrance is rare and positive predictive value approaches $100 \%$. However, the clinical severity of the disease can be difficult to predict due to intra- and interfamilial variability.

\subsection{Negative clinical predictive value}

(Probability not to develop the disease if the test is negative) Assume an increased risk based on family history for a non-affected person. Allelic and locus heterogeneity may need to be considered. 
Index case in that family had been tested:

Approaches 100\%.

Index case in that family had not been tested:

Sequence analysis will detect approximately $95 \%$ of OI patients. ${ }^{8}$

\section{CLINICAL UTILITY}

3.1 (Differential) diagnosis: The tested person is clinically affected (To be answered if in 1.10 'A' was marked)

\subsubsection{Can a diagnosis be made other than through a genetic test?}

\begin{tabular}{lll}
\hline No & $\square$ (continue with 3.1.4) \\
Yes & $\bigotimes$ & \\
& Clinically & \\
& Imaging & $\square$ \\
& Endoscopy & $\square$ \\
& Biochemistry & $\square$ \\
& Electrophysiology & $\square$ \\
& Other (please describe)
\end{tabular}

\subsubsection{Describe the burden of alternative diagnostic methods to the} patient

Prenatally, the severe OI types (not OI type I) can be diagnosed by ultrasound as fractures occur in the prenatal period. Postnatally, the diagnosis OI can be made, based on patient and family history and by clinical and radiological examination (skeletal overview). ${ }^{12,15}$ The burden of clinical and radiological investigations is very low as is the case with imaging by ultrasound in the prenatal period.

The laboratory diagnosis of OI can also be made in most cases by protein analysis: assessing the production (low or abnormal) of collagen type I by cultured skin fibroblasts (skin biopsy). However, not all patients with OI show abnormalities of collagen type I. Furthermore, the underlying genetic cause is not detected, which makes this technique less suitable for prenatal and unsuitable for preimplantation genetic diagnosis. Lastly, blood sampling for genomic sequencing is a less invasive procedure than a skin biopsy. ${ }^{8}$

\subsubsection{How is the cost effectiveness of alternative diagnostic methods to be judged?}

With regard to the diagnosis of OI, prenatal ultrasound (OI types II-IV) and postnatal clinical and radiological examination (all types) are relatively cheap and effective. Protein analysis can be useful as a first-line investigation for countries where reimbursement of molecular analysis is not warranted or as a second line of investigation in cases where molecular analysis did not detect any disease-causing variant within COL1A1 or COL1A2 or any of the so-far identified recessive genes.

3.1.4 Will disease management be influenced by the result of a genetic test?

$\begin{array}{ll}\text { No } & \\ \text { Yes } \begin{array}{l}\text { Therapy } \\ \text { (please describe) } \\ \text { Prognosis } \\ \text { (please describe) }\end{array} & \begin{array}{l}\text { Presently, there is no cure for OI. } \\ \text { (OI type I), to lethal (OI type II), severe (OI type III), } \\ \end{array} \\ & \begin{array}{l}\text { moderately severe (OI type IV and V). DNA analysis can } \\ \text { predict the clinical severity to a certain extent. This makes } \\ \text { the characterization of the genetic defect important. }\end{array}\end{array}$

For dominant OI, haplo-insufficiency mutations in COL1A1 are usually associated with a mild phenotype (OI type I), whereas the more severe phenotypes are usually caused by sequence variants in either COL1A1 or COL1A2 that result in a structural defect of type I collagen. With regard to recessive $\mathrm{OI}$, no patients have been reported with an $\mathrm{OI}$ type I phenotype. However, intra- and interfamilial clinical variability is apparent in $\mathrm{OI}$, increasing the difficulty in predicting the severity of the symptoms.

Management The current management of $\mathrm{OI}$ is interdisciplinary and (please describe) consists of fracture management, surgery and rehabilitation in combination with pharmacological treatment, namely bisphosphonate treatment and supplementation with vitamin D and calcium, if necessary. Studies are being performed to assess whether the underlying genetic cause influences the response to bisphosphonate therapy. Dental treatment, treatment for hearing loss, and prevention of primary (e.g. basilar impression) and secondary (e.g. problems due to general anaesthesia) complications are also the focus of attention. ${ }^{15,16}$

3.2 Predictive Setting: The tested person is clinically unaffected but carries an increased risk based on family history

(To be answered if in 1.10 'B' was marked)

\subsubsection{Will the result of a genetic test influence lifestyle and} prevention?

If the test result is positive (please describe):

Yes, a person might avoid activities with a high chance of trauma with bone fractures as a result. Periodic audiologic evaluation might be considered in adults.

If the test result is negative (please describe):

No.

3.2.2 Which options in view of lifestyle and prevention does a person at-risk have if no genetic test has been done (please describe)? None.

3.3 Genetic risk assessment in family members of a diseased person (To be answered if in 1.10 ' $\mathrm{C}$ ' was marked)

3.3.1 Does the result of a genetic test resolve the genetic situation in that family?

Yes, it confirms the mode of inheritance (autosomal dominant or autosomal recessive) and clarifies the recurrence risk in a future pregnancy in case of non-affected parents with an affected child. The recurrence risk is $25 \%$ for parents of a child affected with recessive OI but $<6 \%$ in dominant OI unless germline mosaicism is demonstrated in the parents. The recurrence risk then depends on the percentage of germline cells containing the mutation and is maximally $50 \%{ }^{8}$ Furthermore, genetic testing is necessary for genetic risk assessment in other relatives.

3.3.2 Can a genetic test in the index patient save genetic or other tests in family members?

Yes, to a certain extent. In the case of dominant OI, the parents of an index patient will always have to be tested, as will subsequent pregnancies. When it concerns a de novo dominant mutation (parents were both tested and did not harbour the mutation) in an index patient with OI type I, other family members do not need to be tested. When it concerns a severe OI phenotype (not OI type I) in the index patient, and the parents were both tested and did not harbour the mutation, 
a recurrence risk in a future pregnancy exists $(<6 \%)$ due to the possibility of germline mosaicism. As such, prenatal diagnosis is possible. Other family members do not need to be tested. However, in the case of an index patient with recessive OI, genetic testing in unaffected relatives is indicated to determine carrier status of recessive OI.

3.3.3 Does a positive genetic test result in the index patient enable a predictive test in a family member?

Yes.

\subsection{Prenatal diagnosis}

(To be answered if in 1.10 'D' was marked)

\subsubsection{Does a positive genetic test result in the index patient enable a prenatal diagnosis? \\ Yes.}

\section{IF APPLICABLE, FURTHER CONSEQUENCES OF TESTING}

Please assume that the result of a genetic test has no immediate medical consequences. Is there any evidence that a genetic test is nevertheless useful for the patient or his/her relatives? (Please describe).

Genetic testing has an additive value in confirming the clinical diagnosis of OI in case of suspicion of NAI but is not always necessary, as the diagnosis can be made based on clinical/radiological examinations whereas a positive genetic test result does not exclude NAI. Genetic testing allows prognostic evaluations to some extent and is the prerequisite for prenatal testing, preimplantation genetic diagnosis and genetic risk calculation in relatives.

\section{CONFLICT OF INTEREST}

The authors declare no conflict of interest.

\section{ACKNOWLEDGEMENTS}

This work was supported by EuroGentest2 (Unit 2: 'Genetic testing as part of health care'), a Coordination Action under FP7 (grant agreement number
261469), EC's 7th Framework Programme (FP7/2007-2013) under grant agreement $\mathrm{n}^{\circ}$ 200754, the GEN2PHEN project and the European Society of Human Genetics.

1 Sillence DO, Senn A, Danks DM: Genetic heterogeneity in osteogenesis imperfecta. J Med Genet 1979; 16: 101-116.

2 Glorieux F, Rauch F, Plotkin $\mathrm{H}$ et al: Type $\mathrm{V}$ osteogenesis imperfecta: a new form of brittle bone disease. J Bone Miner Res 2000; 15: 1650-1658.

3 Warman ML, Cormier-Daire V, Hall C et al: Nosology and classification of genetic skeletal disorders: 2010 revision. Am J Med Genet 2011; 155A: 943-968.

4 Dalgleish R: The human type I collagen mutation database. Nucleic Acids Res 1997; 25: $181-187$.

5 Dalgleish R: The human collagen mutation database 1998. Nucleic Acids Res 1998, 26: 253-255.

6 Semler O, Garbes L, Keupp K et al: A mutation in the 5'UTR of IFITM5 creates an in-frame start codon and causes autosomal-dominant osteogenesis imperfecta type $\mathrm{V}$ with hyperplastic callus. Am J Hum Genet 2012; 91: 349-357.

7 Cho TJ, Lee KE, Lee SK et al: A Single Recurrent Mutation in the 5'-UTR of IFITM5 Causes Osteogenesis Imperfecta Type V. Am J Hum Genet 2012; 91: 343-348.

8 van Dijk FS, Byers PH, Dalgleish $\mathrm{R}$ et al: EMQN best practice guidelines for the laboratory diagnosis of Osteogenesis Imperfecta. Eur J Hum Genet 2012; 20: 11-19.

9 Martínez-Glez V, Valencia M, Caparrós-Martín JA, Aglan M, Temtamy S, Tenorio R: Identification of a mutation causing deficient BMP1/mTLD proteolytic activity in autosomal recessive osteogenesis imperfecta. Hum Mutat 2012; 33: 343-350.

10 van Dijk FS, Pals G, van Rijn RR, Nikkels PG, Cobben JM: Classification of osteogenesis imperfecta revisited. Eur J Med Genet 2010; 53: 1-5.

11 Forlino A, Cabral WA, Barnes AM, Marini JC: New perspectives on osteogenesis imperfecta. Nat Rev Endocrinol 2011; 7: 540-557.

12 Steiner RD, Pepin MG, Byers PH: Osteogenesis Imperfecta, Available at: http://www.ncbi.nlm.nih.gov/bookshelf/br.fcgi?book=gene\&part=oi (accessed 15 May 2012).

13 Cabral WA, Barnes AM, Adeyemo A et al: A founder mutation in LEPRE1 carried by $1.5 \%$ of West Africans and $0.4 \%$ of African Americans causes lethal recessive osteogenesis imperfecta. Genet Med 2012; 14: 543-551.

14 Steiner RD, Pepin MG, Byers PH: Osteogenesis Imperfecta, Available at: http:// www.ncbi.nlm.nih.gov/bookshelf/br.fcgi?book=gene\&part=oi (accessed 30 July 2012).

15 van Dijk FS, Cobben JM, Kariminejad A et al: Osteogenesis Imperfecta: a review with clinical examples. Mol Syndromol 2011; 2: 1-20.

16 Monti E, Mottes M, Fraschini $\mathrm{P}$ et al: Current and emerging treatments for the management of Osteogenesis imperfecta. Ther Clin Risk Manag 2010; 6: 367-381. 